\title{
Heat Pump Efficiencies simulated in Aspen HYSYS and Aspen Plus
}

\author{
Lars Erik Ø $\mathrm{i}^{1} \quad$ Irene Yuste Tirados ${ }^{1}$ \\ ${ }^{1}$ Department of Process, Energy and Environmental Technology, Telemark University College, Norway \\ lars.oi@hit.no
}

\begin{abstract}
The efficiency of a heat pump system has been calculated for varying temperature conditions, refrigerants, simulation programs and thermodynamic models. Evaluated components are ammonia, the chlorofluorocarbons R-11, R-12, R-22 and propane. The equations of state Peng-Robinson (PR) and SoaveRedlich-Kwong (SRK) and the activity model NonRandom-Two-Liquid (NRTL) were used. COP values have been calculated to values between 3 and 9 . Aspen Plus and Aspen HYSYS are evaluated to be powerful tools for heat pump calculations. The calculated differences between heat pump efficiencies with different components at different conditions are reasonable.
\end{abstract}

Keywords: $\quad$ Heat pump, Aspen HYSYS, Aspen Plus, PR, SRK, NRTL

\section{Introduction}

Heat pump technology provides an efficient and sustainable solution for both heating and cooling. A traditional heat pump can be defined as a mechanical compression cycle refrigeration system powered by mechanical energy or electricity. Traditional refrigerants used in heat pumps are ammonia and chlorinated or fluorinated hydrocarbons. Because many of the most used chlorofluorohydrocarbons (CFC's) are ozone-depleting components, development of more environmentally friendly refrigerants like pure hydrocarbons is important (EU, 2009). The efficiency of a heat pump is traditionally measured by the ratio of delivered heat at a high temperature divided by the electricity (or work) input. This is defined as the coefficient of performance (COP).

There are several simulation tools available for the simulation of heat pumps. Traditional process simulation tools like Aspen HYSYS or Aspen Plus are useful because they have data for several components inside the program, and many thermodynamic models like equations of states available. It is of interest to calculate the efficiency of a heat pump system when varying temperature conditions, refrigerants, simulation programs and thermodynamic models. There are few references to such comparisons in the open literature. An example of earlier work on simulation of heat pumps is Domanski and Didion (1985).
This work is based on the Master Thesis work of Irene Yuste Tirados (Tirados, 2015). The background information and the evaluation of the calculation results are extended compared to the content in the Master Thesis report.

\section{Process Description}

The circulation medium (refrigerant) alternates between different pressures and temperatures by the help of a compressor and an expansion valve between the temperatures 25 and 4, 27 and 2 or 27 and $-20{ }^{\circ} \mathrm{C}$. The lowest temperature is the evaporation temperature and the highest is the condensing temperature. The pressures are specified as the saturation pressures at the given temperatures. A simple diagram of the refrigeration circuit is shown in Figure 1.

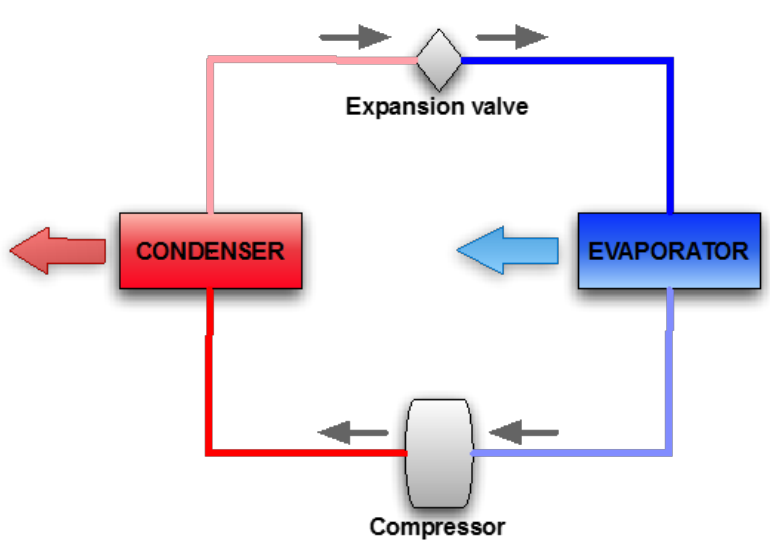

Figure 1. Simple diagram of a heat pump's mechanical compression cycle

In an air to air heat pump, taking heat from a cold environment to a warmer environment, the temperature profiles in the condensation unit and evaporating unit are typically as in Figure 2. The condensing and evaporating temperatures in the figure are 25 and $4{ }^{\circ} \mathrm{C}$. The corresponding outside and outside air temperatures are 9 and $20{ }^{\circ} \mathrm{C}$. In the condenser, the refrigerant coming from the compressor is normally warmer than the condensation temperature and the refrigerant must be cooled down to the condensation temperature in the first part of the condenser. 


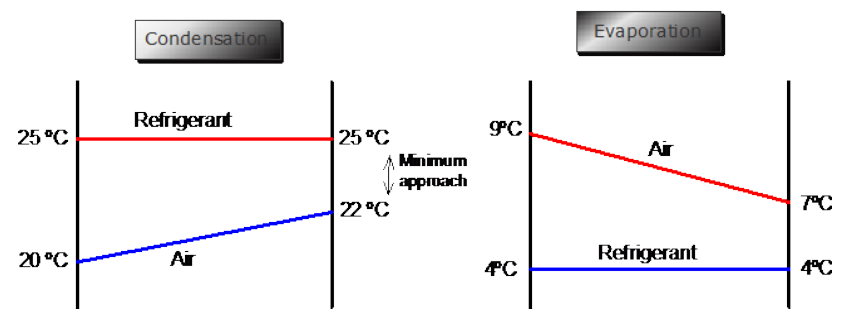

Figure 2. Temperature profiles in the evaporator and condenser for low difference temperature conditions.

The evaluated components are ammonia, R-11, R-12, R-22 and propane. The CFC components are not accepted as refrigerants in heat pumps any longer, but they are relevant because they have been used in many heat pump applications, and new refrigerants components will be compared against the CFC components. The explanations for the abbreviations for the different refrigerant components (the R-numbers) can be found e.g. in an EU report on ozone layer depletion (EU, 2009). The equations of state PengRobinson (PR) and Soave-Redlich-Kwong (SRK) and the activity model Non-Random-Two-Liquid (NRTL) were used in the process simulation programs Aspen HYSYS and Aspen Plus.

The coefficient of performance (COP) is an expression of the efficiency of a heat pump. The coefficient of performance for a heat pump is the ratio of the energy transferred for heating to the input energy used in the process.

$$
\begin{aligned}
& C O P=\frac{Q_{C}}{W} \\
& W=Q_{C}-Q_{E}
\end{aligned}
$$

In equation (1) $\mathrm{Q}_{\mathrm{C}}$ is the heat output from the condenser, $\mathrm{Q}_{\mathrm{E}}$ is the heat input from the evaporator and $\mathrm{W}$ is the power supplied to the compressor. If there is no heat loss the work added equals the difference between the heat output and the heat input.

In this work, it is assumed that the refrigerant loop operates at a specified condensation and evaporation temperature. Because pure components are used in this work, the evaporating and condensing pressures are the saturation pressures. The expansion valve is assumed to operate adiabatically and except of that it is assumed no pressure drops in the circulation loop. The compressor is assumed to have the default adiabatic efficiency of 0.75. A motor or motor efficiency is not included in the calculations, and if a motor efficiency should be included, this would reduce the COP slightly. Version 8.0 of Aspen Plus and Aspen HYSYS have been used.

\section{Thermodynamic Models}

\subsection{General about Property Calculations}

To calculate the temperature $(T)$, pressure $(P$ or $p)$, specific volume $(v)$, heat $(Q)$ or work $(W)$ for different conditions in process simulation programs like Aspen Plus and Aspen HYSYS, there are thermodynamic models, model parameters and calculation procedures available. In other tools for heat pump calculations, thermodynamic property data for the components are directly available as correlations in data banks. These data can be based on direct measurements or they can be based on thermodynamic models.

In the addition to the calculation of energy functions as enthalpy $(H)$ and entropy $(S)$, the key thermodynamic property calculation performed in a simulation is the phase equilibrium. Phase equilibrium is calculated using the fugacity (which is a measure of the tendency of a component to leave its phase). Equilibrium is achieved when the fugacity of the component is equal in all phases. In this work only pure components are treated, so $\varphi_{i}$ is the fugacity of the actual component.

There are two common methods for representing the fugacity coefficients from the phase equilibrium relationship in terms of measurable state variables, the equation of state method and the activity coefficient method as explained in Smith et al. (2005).

Equation of state methods use the various equations of state from chemical engineering thermodynamics to calculate the equilibrium conditions. The two most familiar are PR (Peng and Robinson, 1976) and SRK or Soave-Redlich-Kwong (Soave, 1972). Both of them are cubic equations of state and they only need critical properties and ideal gas enthalpies for each component to calculate all the necessary thermodynamic properties for equilibrium calculations.

An activity coefficient method is a more empirical approach to calculate the equilibrium. This method uses various relationships to calculate the liquid phase activity coefficient and then the fugacity coefficient. A common activity model is NRTL or Non-Random Two Liquid (Renon and Prausnitz, 1968). In Aspen Plus ideal gas enthalpy and heat of vaporization correlations are used to calculate enthalpies. The NRTL model is the same in Aspen HYSYS and Aspen Plus as in the original paper. The results from an activity model are dependent on the parameter values for the components and enthalpy correlations. These parameter values may be different in different program versions.

\subsection{General about Equations of State}

Equation of states are often expressed by critical or reduced temperatures and pressures. The critical temperature $\left(T_{c}\right)$ and pressure $\left(P_{c}\right)$ for a pure component are component characteristics. Reduced properties $\left(T_{r}\right.$ and $P_{r}$ ) are defined as the property divided by the critical 
property. $\mathrm{R}$ is the gas constant. The acentric factor $\omega$ is also a component characteristic which is defined in equation (2).

$$
\omega=-1-\left[\log _{10} P_{r}^{\text {sat }}\right]_{T_{r}=0,7}
$$

A widely used equation of state is the SRK model (Soave, 1972). This model is described by equation (3) with $a$ (the pressure correction parameter), $b$ (the volume correction parameter), $\alpha$ and $m$ defined below.

$$
\begin{gathered}
p=\frac{R T}{v-b}-\frac{a \alpha}{v(v+b)} \\
\left\{a=\frac{0,42748 R^{2} T_{c}^{2}}{p_{c}} \mid b=\frac{0,08664 R T_{C}}{p_{c}}\right\} \\
\alpha=\left[1+m\left(1-T_{r}^{1 / 2}\right)\right]^{2} \\
m=0.48508+1.55171 \omega-0.15613 \omega^{2}
\end{gathered}
$$

These coefficients in the $\mathrm{m}$ equation are from the original work of Soave and can be different in other versions.

A similar equation of state shown in equation (4) was developed by Peng \& Robinson (1976) with more accurate liquid density predictions.

$$
\begin{gathered}
p=\frac{R T}{v-b}-\frac{a \alpha}{v(v+b)+b(v-b)} \\
\left\{a=\frac{0,45724 R^{2} T_{c}^{2}}{p_{c}} \mid b=\frac{0,07780 R T_{C}}{p_{c}}\right\} \\
\alpha=\left[1+m\left(1-T_{r}^{1 / 2}\right)\right]^{2} \\
m=0.37464+1.54226 \omega-0.26992 \omega^{2}
\end{gathered}
$$

The coefficients in the m equation are from the original work of Peng and Robinson.

There are numerous of other equation of state and activity coefficient models, and some of them are available in the Aspen HYSYS and Aspen Plus programs. The PR, SRK and NRTL models are used because they are well-known to be simple and efficient. There are some equations of state which are further developments of the SRK and PR equations of state. One variation is from Peneloux (1982) where a volume correction $c$ is added to every volume in the SRK or PR equations. This is used in the SRK and PR versions in the program Aspen Plus.

In this work, the default parameter values from the Aspen HYSYS and Aspen Plus programs for the PR, SRK and NRTL models are used. There are some small differences between the two programs in the model equations and the parameter values.

\subsection{Aspen HYSYS Property Documentation}

The equations used in Aspen HYSYS are shown in equations (5) to (10). They are from the documentation of an earlier version of the Aspen HYSYS program (Hyprotech, 2003). This documentation is more complete than for the later versions. It is assumed that the equations used are the same also in Aspen HYSYS version 8.0. The equations include mole fractions $\left(x_{i}\right.$ values) and $k_{i j}$ parameters (component interaction coefficients) which are not used in this work with only pure components.

SRK:

$$
\begin{gathered}
p=\frac{R T}{v-b}-\frac{a}{v(v+b)} \\
b=\sum_{i=1}^{N} x_{i} b_{i}, \quad b_{i}=\frac{0,08664 R T_{C}}{p_{c}} \\
a=\sum_{i=1}^{N} \sum_{j=1}^{N} x_{i} x_{j}\left(a_{i} a_{j}\right)^{0,5}\left(1-k_{i j}\right) \\
a_{i}=a_{c i} \alpha_{i}, \quad a_{c i}=\frac{0,42748 R^{2} T_{c}^{2}}{p_{c}} \\
\alpha_{i}=\left[1+m_{i}\left(1-T_{r}^{1 / 2}\right)\right]^{2} \\
m_{i}=0,48+1,574 \omega_{i}-0,176 \omega_{i}^{2}
\end{gathered}
$$

PR:

$$
\begin{gathered}
p=\frac{R T}{v-b}-\frac{a}{v(v+b)+b(v-b)} \\
b=\sum_{i=1}^{N} x_{i} b_{i}, \quad b_{i}=\frac{0,077796 R T_{C}}{p_{c}} \\
a=\sum_{i=1}^{N} \sum_{j=1}^{N} x_{i} x_{j}\left(a_{i} a_{j}\right)^{0,5}\left(1-k_{i j}\right) \\
a_{i}=a_{c i} \alpha_{i}, \quad a_{c i}=\frac{0,457235 R^{2} T_{c}^{2}}{p_{c}} \\
\alpha_{i}=\left[1+m_{i}\left(1-T_{r}^{1 / 2}\right)\right]^{2} \\
m_{i}=0,37464+1,54226 \omega_{i}-0,26992 \omega_{i}^{2}
\end{gathered}
$$

It can be noted that the $m$ coefficients in equation (6) are equal to the original PR coefficients in equation (4). The coefficients in equation (5) are not equal to the original SRK coefficients in equation (3).

\section{Enthalpy and entropy departure calculations}

The principles for the numerical calculation of the enthalpy and entropy calculation (based on residual properties) can be found in Smith et al. (2005). These 
numerical calculations (and also fugacity calculations) may be different in Aspen Plus and Aspen HYSYS.

The enthalpy and entropy departure calculations used in Aspen HYSYS are shown in equations (7) and (8). Z is the compression factor (defined by $R T / P v$ ). Superscript $I D$ is for ideal gas, and subscript 0 is a reference state.

$$
\begin{aligned}
& \frac{H-H^{I D}}{R T}=Z-1-\frac{1}{R T} \int_{\infty}^{v}\left[T\left(\frac{\partial p}{\partial T}\right)_{v}-P\right] d v \\
& \frac{S-S_{0}^{I D}}{T}=\ln Z-\ln \frac{P}{P^{0}}+\int^{v}\left[\frac{1}{R}\left(\frac{\partial p}{\partial T}\right)_{v}-\frac{1}{v}\right] d v
\end{aligned}
$$

\section{Fugacity coefficient}

SRK:

$$
\begin{gathered}
\ln \phi_{i}=-\ln \left(Z-\frac{P b}{R T}\right)+(Z-1) \frac{b_{i}}{b}-\cdots \\
\ldots-\frac{a}{b R T}\left[\frac{1}{a}\left(2 a_{i}^{0,5} \sum_{j=1}^{N} x_{j} a_{j}^{0,5}\left(1-k_{i j}\right)\right)-\frac{b_{i}}{b}\right] \ln \left(1+\frac{b}{V}\right)
\end{gathered}
$$

PR:

$$
\begin{array}{r}
\ln \phi_{i}=-\ln \left(Z-\frac{P b}{R T}\right)-\frac{a}{2^{1,5} b R T}+(Z-1) \frac{b_{i}}{b} \cdot \ldots \\
\ldots \cdot\left[\frac{1}{a}\left(2 a_{i}^{0,5} \sum_{j=1}^{N} x_{j} a_{j}^{0,5}\left(1-k_{i j}\right)\right)-\right. \\
\left.\frac{b_{i}}{b}\right] \ln \left[\frac{V+\left(2^{0,5}+1\right) b}{V-\left(2^{0,5}-1\right) b}\right]
\end{array}
$$

\subsection{Aspen Plus Property Documentation}

The equations used in Aspen Plus are shown in equation (11) to (14). The equations are taken from the documentation of an earlier version of the Aspen Plus program (Aspentech, 2001). As for Aspen HYSYS it is assumed that these are the equations used also for Aspen Plus version 8.0.

SRK:

$$
\begin{gathered}
p=\frac{R T}{v_{m}+c-b}-\frac{a}{\left(v_{m}+c\right)\left(v_{m}+c+b\right)} \\
b=\sum_{i=1}^{N} x_{i} b_{i}, \quad b_{i}=\frac{0,08664 R T_{C}}{p_{c}} \\
a=\sum_{i=1}^{N} \sum_{j=1}^{N} x_{i} x_{j}\left(a_{i} a_{j}\right)^{0,5}\left(1-k_{i j}\right), \quad a_{c i}=\frac{\alpha_{i} 0,42747 R^{2} T_{c}^{2}}{p_{c}} \\
\alpha_{i}=\left[1+m_{i}\left(1-T_{r}^{1 / 2}\right)\right]^{2} \\
c=\sum_{i=1}^{N} x_{i} c_{i}, \quad c_{i}=0,40768\left(\frac{R T_{c}}{P_{c}}\right)\left(0,29441-z_{R A i}\right)
\end{gathered}
$$

These versions of SRK and PR are not equal to the original versions. A volume translation concept and the $c$ parameter introduced by Peneloux and Rauzy (1982) is used to improve the molar liquid volume calculated from the cubic equation of state.

PR:

$$
\begin{gathered}
p=\frac{R T}{\left(c+v_{m}\right)-b}-\frac{a}{\left(v_{m}+c\right)\left(v_{m}+c+b\right)+b\left(v_{m}+c-b\right)} \\
b=\sum_{i=1}^{N} x_{i} b_{i}, \quad b_{i}=\frac{0,07780 R T_{C}}{p_{c}} \\
a=\sum_{i=1}^{N} \sum_{j=1}^{N} x_{i} x_{j}\left(a_{i} a_{j}\right)^{0,5}\left(1-k_{i j}\right) \\
a_{i}=a_{c i} \alpha_{i}, \quad a_{c i}=\frac{\alpha_{i} 0,45724 R^{2} T_{c}^{2}}{p_{c}} \\
\alpha_{i}=\left[1+m_{i}\left(1-T_{r}^{1 / 2}\right)\right]^{2} \\
m_{i}=0,37464+1,54226 \omega_{i}-0,26992 \omega_{i}{ }^{2}
\end{gathered}
$$

$$
c=\sum_{i=1}^{N} x_{i} c_{i}, \quad c_{i}=0,40768\left(\frac{R T_{c}}{P_{c}}\right)\left(0,29441-z_{R A i}\right)
$$

\section{Enthalpy and entropy departure calculations}

The enthalpy and entropy departure calculations use the following equations (13) and (14). Subscript $m$ is for mixture conditions and superscript $i g$ is for ideal gas conditions.

$$
\begin{gathered}
\left(H_{m}-H_{m}^{i g}\right)=-\int_{\infty}^{v}\left(P-\frac{R T}{v}\right) d v-R T \ln \left(\frac{v}{v^{i q}}\right)+\cdots \\
\ldots+T\left(S_{m}-S_{m}^{i g}\right)+R T\left(Z_{m}-1\right) \\
\left(S_{m}-S_{m}^{i g}\right)=-\int_{\infty}^{v}\left[\left(\frac{\partial p}{\partial T}\right)_{V}-\frac{R}{v}\right] d v+R \ln \left(\frac{v}{v^{i q}}\right)
\end{gathered}
$$

\section{Specifications and Calculation Principle}

\subsection{Specifications}

When calculating the COP for a process as in Figure 1 in Aspen HYSYS or Aspen Plus, only a few specifications are necessary. A circulation flow must be specified, but can be specified arbitrarily as e.g. $1 \mathrm{~kg} / \mathrm{h}$. If it assumed that the evaporation and condensing is at saturation conditions, the only necessary specifications 
are the evaporating temperature and condensing temperature.

It is not necessary to calculate the conditions outside the refrigeration circuit to calculate the COP. The air amounts in Figure 2 can be calculated if all the air temperatures are specified. Including an efficiency loss in the compressor motor and including the power needed for air flow in Figure 2 would reduce the calculated COP values slightly.

\subsection{Calculation Principle}

The calculation starts with a pure component flow at the lowest (evaporating) temperature at saturated vapour conditions. Then the vapour is compressed with a default adiabatic efficiency to the pressure which gives condensation at the highest (condensing) pressure. The compressed gas is cooled in a condenser to saturated liquid. Then the liquid is expanded in an expansion valve at adiabatic conditions. The expanded gas/liquid mixture is heated in the evaporator to the original state of saturated vapour. The COP is calculated as the condenser heat divided by the power consumption in the compressor.

\section{Results}

The results are shown in Figures 3 to 7, one figure for each component. In all the figures the results are shown for 3 temperature differences using 3 models and 2 simulation tools.

All the models in both simulation programs show as expected a decrease in COP with increase in temperature difference for all the cases.

For some of the components, the deviations in COP are large for the same model in different programs. These differences are larger than expected.

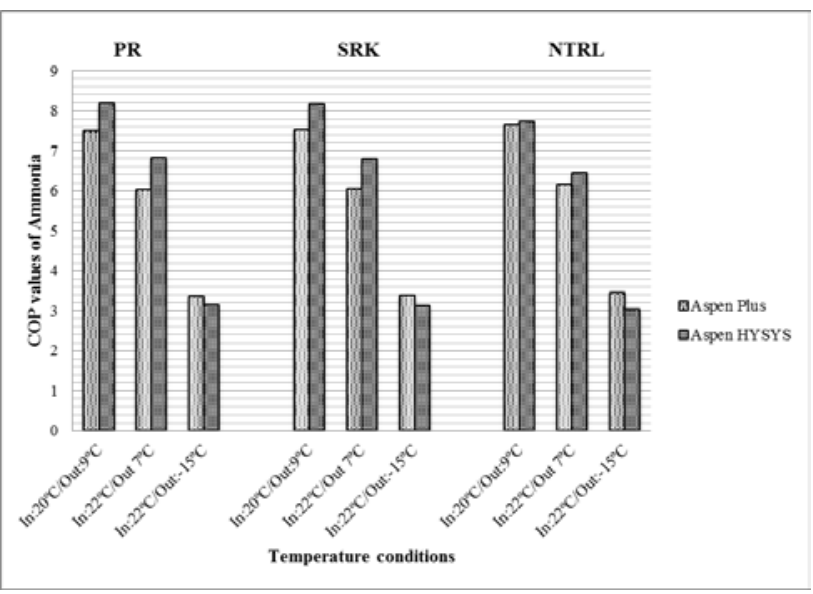

Figure 3. Calculated COP values for ammonia for different temperature conditions, programs and models.
Ammonia is the component with the highest COP (together with R-12) for the lowest temperature difference. As can be seen in Figure 3, all the models in Aspen Plus gave similar COP results for the lowest temperature difference, while Aspen HYSYS gave higher COP values (about 8.2) than Aspen Plus (about 7.5) when using the models PR and SRK.

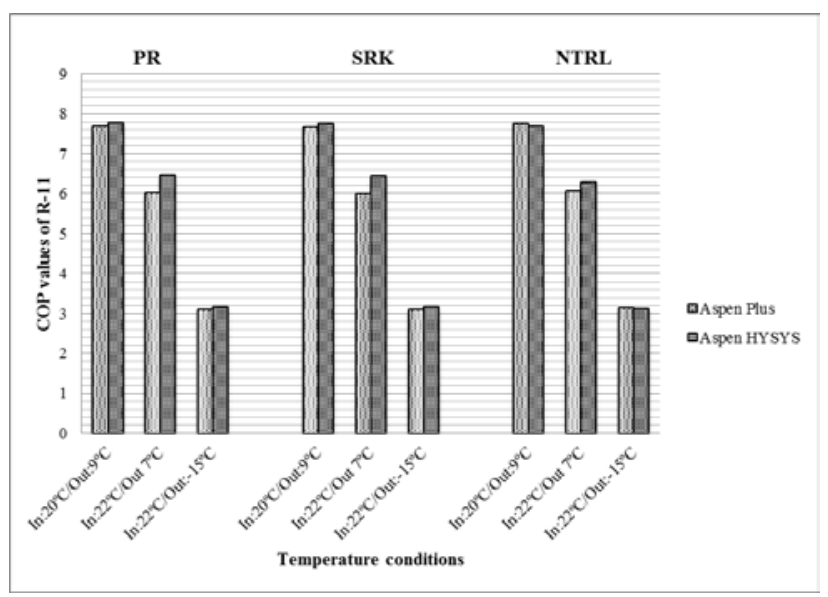

Figure 4. Calculated COP values for R-11 for different temperature conditions, programs and models.

For R-11 there were very few deviations between the models, as seen in Figure 4, but there were slight deviations between COP values calculated with Aspen Plus and Aspen HYSYS. Aspen HYSYS calculated slightly higher COP values for most of the cases.

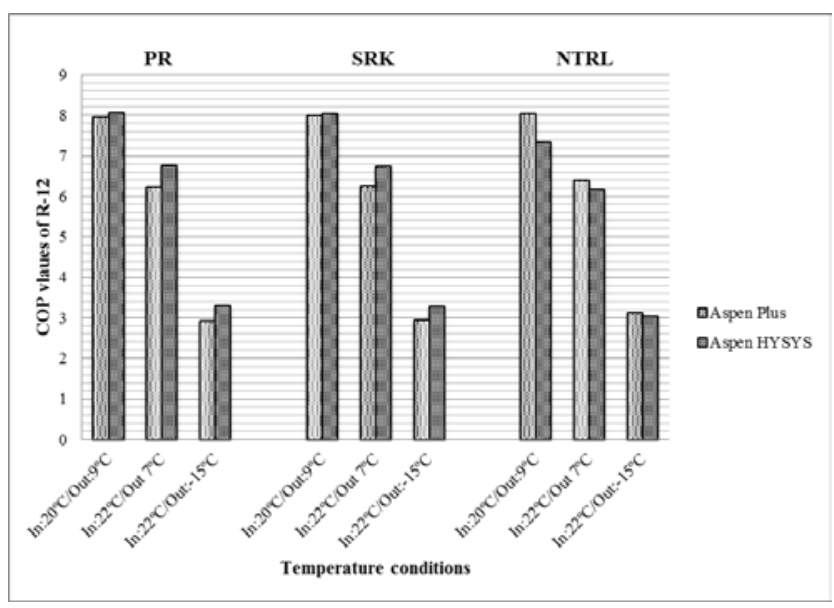

Figure 5. Calculated COP values for R-12 for different temperature conditions, programs and models.

R-12 (together with ammonia) gave the highest COP for the lowest temperature difference. For the low temperature difference, Aspen Plus and Aspen HYSYS gave very similar COP results close to 8.0 as seen in 
Figure 5. Using NRTL, Aspen Plus gave 8.0, while Aspen HYSYS gave a lower value (7.5).

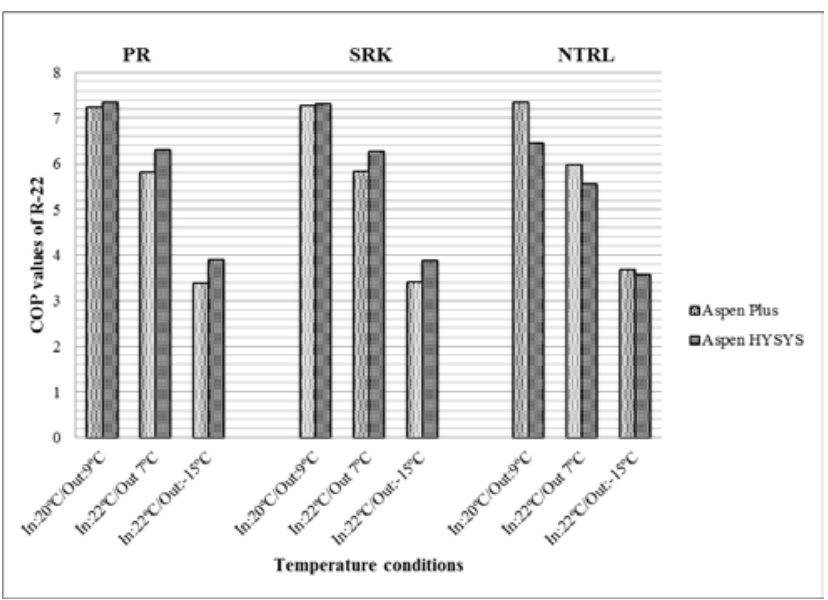

Figure 6. Calculated COP values for R-22 for different temperature conditions, programs and models.

R-22 gave the highest COP values for the highest temperature difference. For the high temperature difference, the COP results in Figure 6 were similar, all between 3.4 and 3.9. The larges deviations were between the simulation programs Aspen Plus and Aspen HYSYS for the PR and SRK models. For PR and SRK Aspen HYSYS calculated the highest COP values. When using the NRTL model, Aspen Plus calculated a slightly higher COP than Aspen HYSYS.

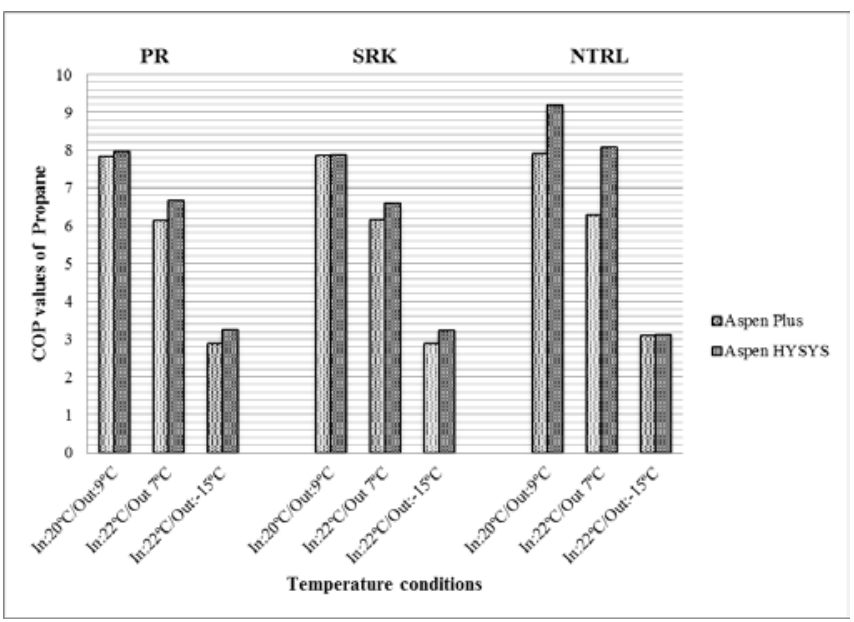

Figure 7. Calculated COP values for propane for different temperature conditions, programs and models.

The COP values in Figure 7 calculated with propane were only slightly less than for the more usual refrigerants. The COP value calculated with Aspen HYSYS using NRTL for the lowest temperature difference was very high (9.2), but all the other COP values for the same conditions was close to 8.0.

\section{Discussion}

The calculated absolute values (COP values) are probably high compared to actual values for heat pumps in operation. Including motor efficiency, heat losses and pressure drops would reduce the calculated COP values. It is however expected that the calculated differences are reasonable. The main purpose of this work is to evaluate these differences.

The models PR and SRK have obtained similar values as expected when calculated in the same simulation program. Both are equations of state using the same parameters, $T_{c}, P_{c}$ and $\omega$.

The model NRTL gave similar results in most of the cases compared to values obtained with PR and SRK, but there were some deviations. As NRTL is not an equation of state method, this difference could be reasonable.

Differences between Aspen Plus and Aspen HYSYS for the same model can be seen. For the PR model slightly higher values are calculated in Aspen HYSYS than in Aspen Plus. However, for the case of NRTL model there is not a clear trend in the deviations.

The explanation of deviations in PR and SRK is also difficult, but some differences in the equations of the models have been found which could be the reason for deviations.

The parameter values of the critical data $\left(T_{c}, P_{c}\right.$ and $\omega)$ were checked to be the same in Aspen Plus and Aspen HYSYS, so this cannot explain the differences. The coefficients in the $m$ equations for SRK are slightly different from Aspen Plus to Aspen HYSYS. The coefficients are however equal for the PR equations, so this can not explain the difference in case of the PR equation.

The most clear difference between the Aspen HYSYS versions and Aspen Plus versions of SRK and PR is that the Aspen HYSYS versions are close to the original SRK and PR versions while the Aspen Plus versions use a volume correction as suggested by Peneloux (1982). These corrections influence the equation of state, the equations for calculation of enthalpy and entropy corrections and the calculation of the fugacity coefficients. This may explain the differences between COP values calculated by Aspen Plus and Aspen HYSYS.

In development of new refrigerants, one trend is to use a mixture of different components. The simulations in Aspen HYSYS and Aspen Plus in this work can be easily extended to multicomponent simulations. The uncertainty in the calculated COP values will probably be slightly higher due to higher uncertainty in the physical property calculation methods. When using the SRK and PR models, it is expected that the thermodynamic properties are calculated quite accurately also for mixtures. It is assumed that calculating heat pumps with a mixture of refrigerants using Aspen HYSYS or Aspen Plus with the SRK or PR 
model will give reasonable results. A reasonable extension of this work is to evaluate the heat pump efficiency for different mixtures at different conditions.

\section{Conclusion}

COP values have been calculated to values between 3 and 9. The highest COP was calculated for the lowest temperature difference. The components giving the highest COP value between the temperatures 22 and 7 ${ }^{\circ} \mathrm{C}$ were ammonia and $\mathrm{R}-12$, and $\mathrm{R}-22$ gave the highest COP between -15 and $22{ }^{\circ} \mathrm{C}$. Propane (which is not a $\mathrm{CFC}$ ) gave slightly lower COP values than the other components.

The differences between the thermodynamic models and the different programs were normally low. However, some differences between Aspen Plus and Aspen HYSYS for the same model were calculated. The calculated deviations between the same models using different programs are difficult to explain. Different equations and model parameters in different programs may explain differences between the same models in different programs.

Aspen Plus and Aspen HYSYS are powerful tools for heat pump calculations. The calculated differences between heat pump efficiencies with different components at different conditions are thought to be reasonable.

\section{Symbol List}

$\begin{array}{lll}\text { Symbol } & \text { Description } & \text { Unit } \\ \text { a } & \text { Parameter in PR and SRK } & {\left[\mathrm{J} \cdot \mathrm{m}^{3} / \mathrm{mol}^{2}\right]} \\ \text { b } & \text { Parameter in PR and SRK } & {\left[\mathrm{m}^{3} / \mathrm{mol}^{2}\right]} \\ \text { c } & \text { Parameter in PR and SRK } & {\left[\mathrm{m}^{3} / \mathrm{mol}\right]} \\ \text { G } & \text { Specific Gibbs Free Energy } & {[\mathrm{J} / \mathrm{mol}]} \\ \text { H } & \text { Specific enthalpy } & {[\mathrm{J} / \mathrm{mol}]} \\ \text { k } & \text { Binary parameter in PR and SRK } & {[-]} \\ \text { m } & \text { Parameter in PR and SRK } & {[-]} \\ \text { P,p } & \text { Pressure } & {\left[\mathrm{N} / \mathrm{m}^{2}\right]} \\ \text { Q } & \text { Heat } & {[\mathrm{J} / \mathrm{s}]} \\ \text { R } & \text { Gas constant } & {[\mathrm{J} /(\mathrm{mol} \cdot \mathrm{K})]} \\ \text { S } & \text { Specific entropy } & {[\mathrm{J} /(\mathrm{mol} \cdot \mathrm{K})]} \\ \text { T } & \text { Temperature } & {[\mathrm{K}],\left[{ }^{\circ} \mathrm{C}\right]} \\ \text { v } & \text { Molar volume } & {\left[\mathrm{m}^{3} / \mathrm{mol}\right]} \\ \text { W } & \text { Work } & {[\mathrm{J} / \mathrm{s}]} \\ \text { x } & \text { Mole fraction } & {[-]} \\ Z & \text { Compressibility factor } & {[-]} \\ \alpha & \text { Parameter in PR and SRK } & {[-]} \\ \varphi & \text { Fugacity coefficient } & {[-]} \\ \omega & \text { Acentric factor } & {[-]}\end{array}$

$\begin{array}{ll}\text { Subscripts/superscripts: } \\ \text { C } & \text { Condenser } \\ \text { c } & \text { Critical } \\ \text { E } & \text { Evaporator } \\ \text { i } & \text { General component } \\ \text { ID } & \text { Ideal } \\ \text { ig } & \text { ideal gas } \\ \text { j } & \text { General component } \\ \text { m } & \text { mixture } \\ \text { r } & \text { Reduced } \\ \text { sat } & \text { Saturated } \\ 0 & \text { Reference state }\end{array}$

\section{References}

AspenTech: Aspen Physical Property System 11.1, 2001.

P.A. Domanski and D.A. Didion: Simulation of heat pump operating with nonazeotropic mixture. ASHRAE Technical Data Bulletin, 91(3):54, 1985.

EU Parliament: Regulation (EC) No 1005/2009 on Substances that Deplete the Ozone Layer, 2009.

Hyprotech: HYSYS Simulation Basis, 2003.

A. Peneloux, E. Rauzy and R. Freze: A Consistent Correction for Redlich-Kwong-Soave Volumes: Fluid Phase Equilibria, 8:7-23, 1982.

D.Y. Peng and D.B. Robinson: A New Two-Constant Equation of State. Industrial and Engineering Chemistry Fundamentals, 15(1):59-64, 1976.

H. Renon and J.M. Prausnitz: Local compositions in Thermodynamic Excess Functions for Liquid Mixtures. A.I.Ch.E. Journal, 14(1):135-144, 1968.

J.M. Smith, H.C. Van Ness and M.M. Abbott. Introduction to Chemical Engineering Thermodynamics, $7^{\text {th }}$ ed., McGraw Hill, 2005.

G. Soave: Equilibrium Constants from a Modified RedlichKwong Equation of State. Chemical Engineering Science, 27:1197-1203, 1972.

I.Y. Tirados: Heat pump efficiencies simulated with Aspen HYSYS and Aspen Plus. Master Thesis, Telemark University College, 2015. 